\title{
Spinal subdural hematoma and ankylosing spondylitis: case report and review of literature
}

\author{
Darian R. Esfahani $\mathbb{1}^{1} \cdot$ Harsh P. Shah $\mathbb{1}^{1} \cdot$ Mandana Behbahani ${ }^{1} \cdot$ Gregory D. Arnone ${ }^{1} \cdot$ Ankit I. Mehta $^{1}$
}

Received: 28 December 2017 / Revised: 21 January 2018 / Accepted: 26 February 2018

(c) International Spinal Cord Society 2018

\begin{abstract}
Introduction Spinal subdural hematomas are rare, disabling hemorrhages. Ankylosing spondylitis (AS) is a relatively common inflammatory condition of the spine that can progress to a fragile, unstable fusion vulnerable to fracture. While spinal epidural hematomas have been described, subdural hematomas to date have not been reported in AS. In this report, we describe the unique case of a patient on warfarin with AS who developed a spinal subdural hematoma and fracture in the absence of trauma. We then discuss the pathogenesis, presentation, prognosis, and management strategies for this unique diagnosis.

Case presentation A 60-year-old man with recent AS diagnosis and atrial fibrillation on warfarin presented with $96 \mathrm{~h}$ of low back pain and $24 \mathrm{~h}$ of leg weakness and urinary retention. CT imaging revealed a bamboo spine and fracture of the posterior elements at L4, while MR revealed a hematoma with thecal sac compression. The warfarin was reversed and the patient taken to the operating room; on laminectomy, however, no hematoma was encountered. The patient then underwent intraoperative ultrasound, durotomy, and evacuation of a thick subdural hematoma, followed by posterior fusion.

Discussion This case represents the first report of an AS patient who developed a subdural hematoma requiring evacuation. Although rare, the clinician should maintain a broad differential and be familiar with this unique pathology, particularly in high-risk patients, such as those with suspected fractures or on warfarin. In patients with back pain and myelopathic symptoms, rapid diagnosis followed by prompt evacuation allows for the best opportunity for neurologic recovery.
\end{abstract}

\section{Introduction}

Acute spinal subdural hematomas are dangerous, potentially disabling hemorrhages [1]. While epidural hematomas are a relatively common cause of symptoms after trauma or surgery [2], subdural hematomas, by contrast, are very rare. Acute spinal subdural hematomas have been reported following lumbar punctures [3], spinal [4] and cranial surgery [5], and trauma [6], with spontaneous hemorrhages relatively uncommon. The few spontaneous hemorrhages reported in the literature include patients with bleeding disorders, those on anticoagulant therapy [1], and underlying lesions, such as vascular malformations or tumors [7].

Ankit I. Mehta

ankitm@uic.edu

1 Department of Neurosurgery, University of Illinois at Chicago, Chicago, IL, USA
Ankylosing spondylitis (AS) is a common, progressive inflammatory condition of the axial skeleton that typically presents initially with back pain, but later is characterized by stiffness, loss of mobility, and proliferative changes leading to ankylosis, syndesmophytes, and fusion [8]. Patients with chronic AS exhibit decreased bone density and are at elevated risk of fractures and spinal cord injury with minimal trauma [9]. Patients with AS are further vulnerable to the development of epidural hematomas, leading to elevated risk of spinal cord compression and myelopathy [10].

While spinal epidural hematomas have been described, subdural hematomas are very rare, and to date have not been reported in the AS population. In this report, we describe the case of a unique patient with AS on warfarin who developed a spinal subdural hematoma and vertebral fracture in the absence of trauma. We then discuss the pathogenesis, presentation, prognosis, and management strategies for this unique diagnosis. 


\section{Case presentation}

\section{History and presentation}

A 60-year-old male with recent diagnosis of AS and multiple comorbidities including morbid obesity, chronic renal disease, deep venous thrombosis, and atrial fibrillation on warfarin presented to the emergency room with $96 \mathrm{~h}$ of severe low back pain and $24 \mathrm{~h}$ of bilateral lower extremity weakness and urinary retention.

Prior to the onset of symptoms, the patient reported getting out of bed and feeling a sudden "pop" followed by jarring pain in the lower back. Although walking was challenging secondary to pain, the patient denied any initial neurological deficits at the time of symptom onset. Over the next $72 \mathrm{~h}$, the patient developed symptoms of urinary retention and bilateral lower extremity numbness and weakness, for which he presented to an outside hospital. Computed tomography (CT) imaging at the time revealed a bamboo appearing spine with autofusion and fracture of the posterior elements at the L4 level (Fig. 1) and follow-up magnetic resonance imaging (MRI) revealed a suspected epidural hematoma extending from T12-S1 with compression of the thecal sac (Fig. 2). The patient was subsequently transferred to our institution for cauda equina syndrome. On arrival, the patient was assessed using the ASIA/ISCoS International Standards for Neurological Classification of Spinal Cord Injury (ISNCSI) [11] and found to be ASIA D with strength of 4/5 and incomplete, decreased sensory loss diffusely throughout the lower extremities bilaterally.

\section{Surgical evacuation}

The patient was found to have an elevated international normalized ratio (INR) of 4.5, which was reversed emergently with prothrombin complex concentrate before the patient was taken to the operating room. The patient then underwent L2-S1 laminectomy; on removal of the lamina and ligamentum flavum, however, no epidural hematoma was encountered. The thecal sac was inspected and noted to be full, and an intraoperative ultrasound was performed which was suggestive of subdural hematoma. The patient subsequently underwent durotomy from L2-S1 and a thick, subdural hematoma was encountered (Fig. 3). The hematoma was systematically evacuated with forceps and saline irrigation, and the thecal sac was irrigated rostral and caudal to the durotomy site using an angiocatheter to remove any residual blood. After all visible blood was removed, the thecal sac was closely examined and no source of bleeding was identified. With the cauda equina and exiting nerve roots adequately decompressed, the dura was closed and the fracture addressed with L2-S1 spinal fusion. Throughout the case, somatosensory and motor evoked potentials were stable and the patient awoke with an improved neurologic examination with strength and sensory improvement.

\section{Postoperative course}

The patient was transferred to the neurosurgical stepdown unit and $48 \mathrm{~h}$ postoperatively underwent spinal angiogram, which was negative for the source of the subdural hemorrhage. While the patient's subsequent hospital stay was significant for delirium, newly diagnosed sleep apnea, and difficulty obtaining adequate pain control, the patient was ultimately discharged after 13 days to a rehabilitation facility. On most recent follow-up at 6 months, the patient was clinically well, had satisfactory instrumentation, and exhibited intact strength, sensation, urinary, and bowel function (ASIA E).
Fig. 1 Preoperative CT lumbar spine. Sagittal (a) and axial (b) preoperative imaging was consistent with fusion of the posterior elements of the lumbar spine and an acute fracture (arrows)

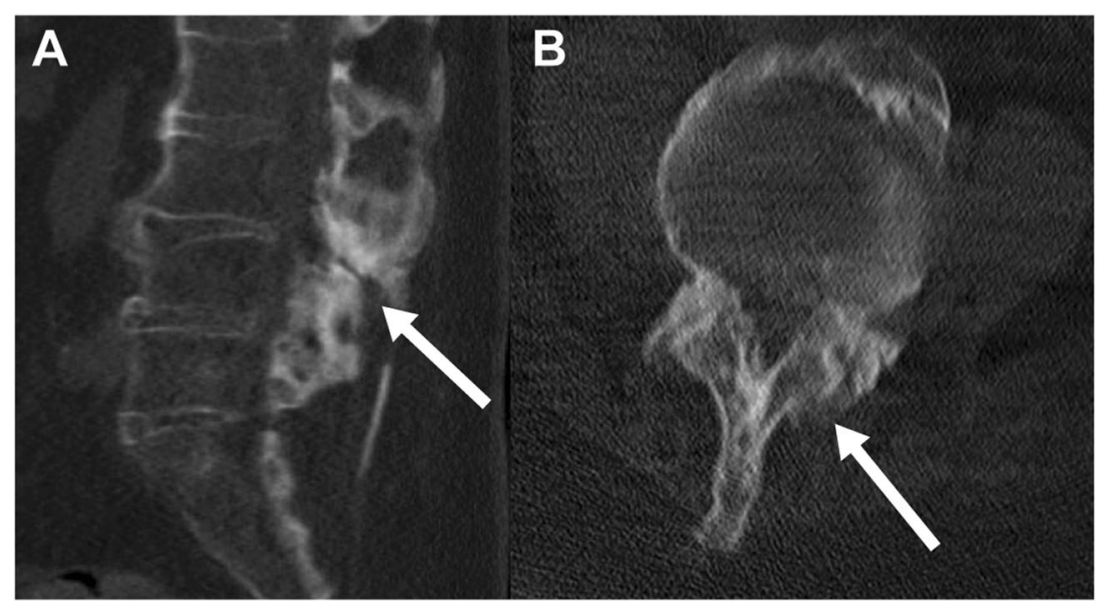


Fig. 2 Preoperative MRI lumbar spine. Sagittal (a) and axial (b) preoperative imaging revealed a subdural hemorrhage with fluid-fluid level (arrows)

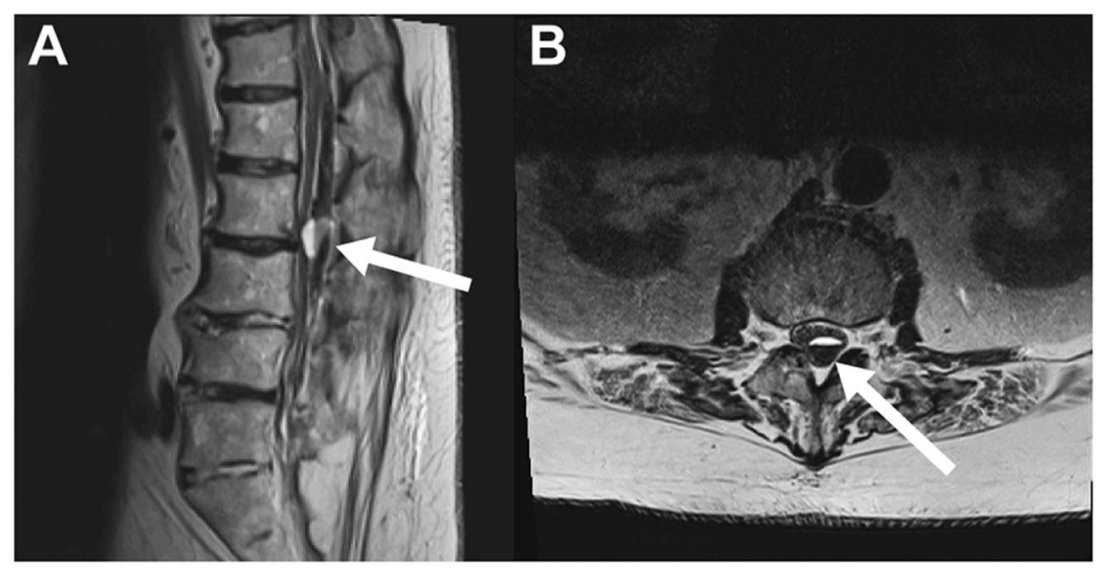

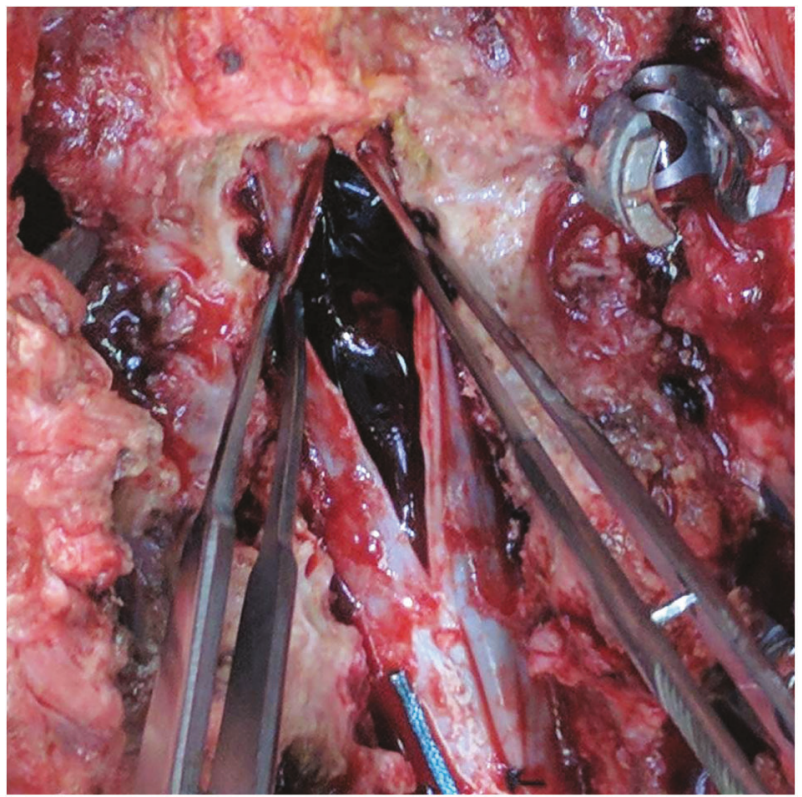

Fig. 3 Spinal subdural hematoma, intraoperative image. On exposure of the thecal sac, no hemorrhage was immediately visible. On dural opening, a thick blood clot, pictured, was visualized and evacuated

\section{Discussion}

This case represents the first report of a patient with AS on warfarin with a nontraumatic fracture who developed a subdural hematoma requiring evacuation. Although the exact etiology is unclear, it is likely that the fracture from AS ruptured a subdural vessel, which, combined with a supratherapeutic INR on warfarin, allowed for formation of a large, symptomatic subdural clot. Although spinal subdural hemorrhages are rare, their presentation can be similar to more common hemorrhages, such as epidural hematomas. As a consequence, consideration of medical conditions, such as AS, and maintaining a broad differential is essential. In addition, intraoperative aids such as ultrasound can guide surgical management and exploration during unusual case presentations.

\section{Ankylosing spondylitis}

AS is a common inflammatory spine disease associated with axial arthritis, low back pain, and eventually progressing to ankylosis, decreased mobility, and a fragile spinal fusion [8]. AS generally presents at a young age, effects men twice as often as women, and has a prevalence between $0.1 \%$ and $1.4 \%$ in patients of European descent [8], with increased risk among patients who are positive for HLA B27 [12].

While the precise cause of AS development remains unknown, recent research suggests that a potential mechanism is the interaction between bacteria or other micro-organisms and HLA B27, causing an inflammatory response and ultimately new bone formation [13]. Ossification of the annulus fibrosis, formation of syndesmophytes, and fusion of the vertebral body margins provide an appearance of a single, fused vertebral column, which, on anterior-posterior view, resembles a bamboo stem, giving the name "bamboo spine" [14]. This fusion is not stable, however, and is exquisitely vulnerable to fracture with minimal, sometimes overlooked trauma, as was the case in this patient. Epidural hematomas have been described in patients after injury with AS, with an early series describing epidural hematomas in four of eight AS patients presenting with acute cervical injury [15]. The exact mechanism of hemorrhage in AS is unknown, but likely arises from bleeding from the overlying fractured bone, epidural soft tissue, or a disrupted venous plexus, and is facilitated by anticoagulation [15].

Regardless of cause, spinal hemorrhages after AS typically present with rapid onset of symptoms, including neurologic deficits, secondary to compression [10]. In this case, the patient's symptoms evolved in a subacute manner, with pain at $96 \mathrm{~h}$ and weakness $24 \mathrm{~h}$ prior to presentation, respectively, suggesting a gradually expanding hematoma in the presence of coagulopathy. The lumbar location in this case is also significant, with the mobile fibers of the cauda equina capable of sustaining a greater hematoma volume 
than the spinal cord proper. Finally, the unusual subdural location of the hemorrhage may have allowed for greater distribution of blood within the thecal sac, delaying the onset of compression capable of causing a neurologic deficit. Irrespective of hemorrhage location, however, upon onset of neurologic deficits, outcomes are best with rapid decompression, ideally within $12 \mathrm{~h} \mathrm{[16].}$

\section{Spinal subdural hematomas}

While symptomatic spinal hemorrhages are uncommon in themselves, spinal subdural hematomas are even more rare. In a large meta-analysis reviewing 613 spinal hematomas, subdural hemorrhages made up only $4 \%$ of cases. Epidural hemorrhages, by contrast, represented $76 \%$ of the sample, while subarachnoid bleeds represented $16 \%$ [17]. While the precise reason for their rarity is unclear, some authors attribute the low rate of subdural bleeds to the small "capillary slit" of the subdural space, compared to larger subarachnoid and epidural spaces [17].

The specific etiology of spinal subdural hemorrhages remains unknown. A proposed mechanism is that trauma, such as this case, or increased intrathoracic pressure can cause rupture of the subarachnoid veins, with blood initially spilling into the cerebrospinal fluid space, and then later extending into the subdural space by diffusion [17]. Other authors point to large veins, visible on some angiographic studies, in the dorsolateral dura that are vulnerable to injury or rupture, causing subdural hemorrhage [3]. Regardless of etiology, however, the presence of anticoagulants is an important risk factor for the development and expansion of spinal hemorrhages, with previous reports demonstrating spontaneous spinal hematomas in the presence of rivaroxiban [18] and warfarin [19].

A recent meta-analysis by de Beer et al. confined to spinal subdural hematomas identified a mean age of $59.5 \mathrm{y}$, the age of the patient in this case, with a slight female predominance (57\%) [20]. Spinal subdural hematomas are observed most often in the thoracic spine, with predominately lumbar or lumbosacral subdural bleeds representing only $9 \%$ of cases [20]. The most common initial presentation in spinal subdural hematomas is motor deficit, seen in $89 \%$, followed by pain, in $84 \%$.

Spinal hemorrhages are most frequently idiopathic in nature, followed by hemorrhages secondary to anticoagulation, such as this patient [17, 20]. Vascular malformations, including arteriovenous malformations and dural venous fistulas, are also associated with spinal hemorrhages, followed by procedures, trauma, and fractures, also observed in this case [17]. Less common causes reported in the literature have included vasculitides, like Churg-Strauss syndrome or Wegener granulomatosis [20], and vigorous back massage [21].
Spinal subdural hemorrhages, in general, have a gradual onset, with a mean time from ictus to symptoms of $72 \mathrm{~h}$, similar to the $96 \mathrm{~h}$ in this case. Epidural hematomas, by contrast, had a mean time of $16 \mathrm{~h}$ to symptom onset [17]. Approximately $30 \%$ of patients with spinal subdural bleeds requiring surgery make a full recovery, with another $20 \%$ able to ambulate with a walking aid [20], evidence of the high morbidity for this diagnosis.

\section{Management strategies for the spinal subdural hematoma patient}

Spinal subdural hematomas, while rare, are a discrete pathologic identity that should remain on the differential of the treating clinician. We recommend that special consideration be given to patients with AS who present with acute onset of back pain and either acute or subacute myelopathic symptoms, including weakness, sensory loss, bowel, or bladder symptoms. Additional concern should further be applied to patients on anticoagulation. Expedient CT imaging should be obtained first to identify fractures, which are very common in this population, followed by an MRI to assess for spinal cord or nerve root compression.

Fluid-fluid levels extending across multiple spinal segments are highly suggestive of spinal hemorrhage, and should be considered even in the absence of trauma or fracture. Distinction between epidural and subdural hemorrhages can be aided by identifying whether fluid is above or below the dural surface (Fig. 2) and whether the onset of myelopathic symptoms is acute or subacute for epidural and subdural hemorrhages, respectively. Subarachnoid hemorrhages can further be distinguished by presence of radicular symptoms, spinal rigidity, and meningitic signs. Once diagnosed, prompt evacuation is recommended in patients with neurologic deficits. Intraoperatively, subdural exploration, aided by ultrasound, should be considered if hemorrhage is suspected and not found after laminectomy. Stabilization should also be considered in AS patients or those with unstable fractures.

\section{Conclusions}

This is the first reported case of a spinal subdural hematoma in a patient on warfarin with AS and a nontraumatic fracture. Although rare, the clinician or surgeon should maintain a broad differential and be familiar with this unique pathology, particularly in high-risk patients, such as those with AS or on anticoagulation. In patients with back pain and myelopathic symptoms, rapid diagnosis followed by prompt evacuation allows for the best opportunity for neurologic recovery. 


\section{Compliance with ethical standards}

Conflict of interest The authors declare that they have no conflict of interest.

\section{References}

1. Russell NA, Benoit BG. Spinal subdural hematoma. A review. Surg Neurol. 1983;20:133-7.

2. Aono H, Ohwada T, Hosono N, Tobimatsu H, Ariga K, Fuji T, et al. Incidence of postoperative symptomatic epidural hematoma in spinal decompression surgery. $\mathrm{J}$ Neurosurg Spine. 2011;15:202-5.

3. Edelson RN, Chernik NL, Posner JB. Spinal subdural hematomas complicating lumbar puncture: occurrence in thrombocytopenic patients. Arch Neurol. 1974;31:134-7.

4. Nagamoto Y, Takenaka S, Aono H. Postoperative spinal subdural lesions following lumbar spine surgery: prevalence and risk factors. Asian Spine J. 2017;11:793-803.

5. Kokubo Rinko, Kim Kyongsong, Mishina Masahiro, Isu Toyohiko, Kobayashi Shiro, Yoshida Daizo, et al. Prospective assessment of concomitant lumbar and chronic subdural hematoma: is migration from the intracranial space involved in their manifestation? J Neurosurg Spine. 2014;20:157-63.

6. Hung KS, Lui CC, Wang CH, Wang CJ, Howng SL. Traumatic spinal subdural hematoma with spontaneous resolution. Spine. 2002;27:E534-8.

7. Han PatrickP, Theodore Nicholas, Porter RandallW, Detwiler PaulW, Lawton MichaeLT, Robert, Spetzler F. Subdural hematoma from a Type I spinal arteriovenous malformation. J Neurosurg Spine. 1999;90:255-7.

8. Braun J, Sieper J. Ankylosing spondylitis. Lancet. 2007;369:1379-90.

9. Ghozlani I, Ghazi M, Nouijai A, Mounach A, Rezqi A, Achemlal $\mathrm{L}$, et al. Prevalence and risk factors of osteoporosis and vertebral fractures in patients with ankylosing spondylitis. Bone. 2009; $44: 772-6$
10. Wu CT, Lee ST. Spinal epidural hematoma and ankylosing spondylitis: case report and review of the literature. J Trauma. 1998;44:558-61.

11. Kirshblum SC, Burns SP, Biering-Sorensen F, Donovan W, Graves DE, Jha A, et al. International standards for neurological classification of spinal cord injury (Revised 2011). J Spinal Cord Med. 2011;34:535-46.

12. Khan MA. Epidemiology of HLA-B27 and arthritis. Clin Rheumatol. 1996;15 Suppl 1:10-2..

13. Hacquard-Bouder C, Ittah M, Breban M. Animal models of HLAB27-associated diseases: new outcomes. Joint Bone Spine. 2006;73:132-8.

14. Fox MarkW, Onofrio BurtonM, John, Kilgore E. Neurological complications of ankylosing spondylitis. J Neurosurg. 1993;78:871-8.

15. Bohlman HH. Acute fractures and dislocations of the cervical spine. An analysis of three hundred hospitalized patients and review of the literature. $\mathrm{J}$ Bone Joint Surg Am. 1979;61:1119-42.

16. Lawton MT, Porter RW, Heiserman JE, Jacobowitz R, Sonntag VK, Dickman CA. Surgical management of spinal epidural hematoma: relationship between surgical timing and neurological outcome. J Neurosurg. 1995;83:1-7.

17. Kreppel D, Antoniadis G, Seeling W. Spinal hematoma: a literature survey with meta-analysis of 613 patients. Neurosurg Rev. 2003;26:1-49.

18. Castillo JM, Afanador HF, Manjarrez E, Morales XA. Nontraumatic spontaneous spinal subdural hematoma in a patient with non-valvular atrial fibrillation during treatment with rivaroxaban. Am J Case Rep. 2015;16:377-81.

19. Sandvig A, Jonsson H. Spontaneous chronic epidural hematoma in the lumbar spine associated with Warfarin intake: a case report. SpringerPlus. 2016;5:1832.

20. de Beer MH, Eysink Smeets MM, Koppen H. Spontaneous spinal subdural hematoma. Neurologist. 2017;22:34-9.

21. Maste P, Paik S-H, Oh J-K, Kim Y-C, Park M-S, Kim T-H, et al. Acute spinal subdural hematoma after vigorous back massage: a case report and review of literature. Spine. 2014;39:E1545-8. 